\title{
On the Maintenance of the Axisymmetric Part of the Flow in the Atmosphere1)
}

\author{
By JACQUES DeRome ${ }^{2}$ ) and A. WIIN-NIELSEN ${ }^{3}$ )
}

\begin{abstract}
Summary - The maintenance of the axisymmetric component of the flow in the atmosphere is investigated by means of a steady-state, quasi-geostrophic formulation of the meteorological equations. It is shown that the meridional variations in the time-averaged axisymmetric variables can be expressed as the sum of three contributions, one being due to the eddy heat transport, another to the eddy momentum transport, and a third to the convective-radiative equilibrium temperature which enters the problem through the specification of a Newtonian form of diabatic heating. The contributions by the large scale eddies are evaluated through the use of observed values for the eddy heat and momentum transports.

The contributions from each of the three forcing mechanisms to the temperature and zonal wind fields are investigated individually and found to be of about equal importance. The sum of the three contributions are also presented for the temperature, the zonal wind, the stream function associated with the mean meridional circulation and the corresponding vertical motion. Although the results fail to reproduce the main observed features of the lower stratosphere, they are found to be in good agreement with observations in the middle latitude troposphere. At any pressure level, for example, the computed mean zonal wind has a jet-like profile and the axis of the jet is found to slope to the south with height, as observed in the atmosphere.
\end{abstract}

\section{Introduction}

A great deal of work has been done in the last two decades on the problem of determining how the axisymmetric part of the flow in the atmosphere is maintained against friction. A number of studies used the approach which consists in measuring, or computing from meteorological observations, the magnitude of the various energy sources and sinks contributing to the energy budget of the zonally averaged flow. Among these investigations we find, for example, those of STARR and WHITE [22],4) Houghton [8] and Wirn-Nielsen [28]. Several other references can be found in REITER [17]. It has become clear from these studies that while radiative processes provide the fundamental mechanism through which the atmosphere as a whole

1) Based in part on a thesis submitted by the first author as partial fulfilment of the requirements for the Ph.D. degree at the University of Michigan. - Publication No. 194 from the Department of Meteorology and Oceanography, The University of Michigan.

2) Meteorological Service of Canada, Montreal, Quebec, Canada.

3) Department of Meteorology and Oceanography, The University of Michigan, Ann Arbor, Michigan, U.S.A.

4) Numbers in brackets refer to References, page 184. 
receives its energy, the large-scale baroclinic eddies play a vital role in redistributing this energy in the north-south direction, thus affecting the structure of the axisymmetric component of the flow.

The other approach to the axisymmetric regime problem has consisted in developing and investigating various models of the atmosphere. In some cases (e.g. Mintz [14], MANabe et al. [11], Washington and Kasahara [24]) rather sophisticated general circulation models are integrated numerically over extended periods of time to obtain statistics regarding, in particular, the way in which various physical factors influence the zonally averaged flow. In other cases the equations for the axisymmetric part of the variables are used $a b$ initio and the sources and sinks of energy are either specified or parameterized in terms of the axisymmetric variables themselves so that a closed system of equations can be obtained. Studies of this type include, for example, those of Kuo [9], Adem [1], Williams and Davies [31] and Saltzman [19]. For convenience these papers will be denoted by the letters K, A, $\mathrm{W}-\mathrm{D}$ and $\mathrm{S}$, respectively. In these the atmospheric meridional eddy heat transport has either been parameterized in terms of the axisymmetric temperature field $T_{z}$ $(\mathrm{A}, \mathrm{W}-\mathrm{D}, \mathrm{S})$ or considered qualitatively $(\mathrm{K})$ and the meridional eddy momentum transport has been neglected (A, S), parameterized in terms of $T_{z}(\mathrm{~W}-\mathrm{D})$ or computed from observations for the purposes of a qualitative discussion (K). In most cases the zonally averaged diabatic heating was parameterized and in one study (W-D) some computations were also performed with a prescribed distribution of the diabatic heating.

In the present study an investigation of the axisymmetric state problem is made by means of a steady state model in which the diabatic heating and the friction mechanisms are parameterized while the horizontal eddy heat and momentum transports are taken to be known from observations. The model therefore differs from those of $\mathrm{A}$ and $\mathrm{S}$ in that, in particular, it includes the effects of the eddy momentum transport which were neglected by the latter. In addition these two studies investigated the meridional variations of vertically averaged variables whereas here the vertical variations will also be considered. One important difference with the model used in W-D lies in the way in which the effects of the eddy heat and momentum transports are taken into account. It is clear that while the parameterization of these effects, as done in W-D, offers a promising avenue of research, it is also important to examine how the large-scale eddies in the atmosphere actually affect the axisymmetric components of the meteorological variables by using transport data computed from observations. This, in essence, is the main objective of the present study.

In sections 2 and 3 the model equations are presented and the nature of the data used is discussed. The effects of the eddy heat and momentum transports, the friction mechanism and the diabatic heating on the temperature field are described in section 4 , followed by a discussion of the computed mean meridional circulation in section 5 . The effects of the various forcing mechanisms on the zonal wind are then presented in section 6 and finally a summary of the main results is given in section 7 . 


\section{The model equations}

Our treatment of the axisymmetric regime is based on a quasi-geostrophic formulation of the hydrodynamic equations written in terms of a spherical coordinate system where $\lambda, \varphi$, and $p$ are the longitude, latitude, and pressure, respectively. The time dependence is removed from the equations by averaging them with respect to time over a period of one year and assuming that all yearly averaged quantities are time independent. If we use a bar over a symbol to designate its annual mean value we can write the time-averaged first equation of motion as

$$
\overline{\frac{u}{a \cos \varphi} \frac{\partial u}{\partial \lambda}}+\frac{\bar{v} \frac{\partial u}{a}}{\partial \varphi}-\frac{\overline{u v \tan \varphi}}{a S}=\frac{1}{a \cos \varphi} \frac{\partial \bar{\phi}}{\partial \lambda}+f \bar{v}+\bar{F}
$$

where $u$ and $v$ are the zonal and meridional components of the wind velocity respectively, $a$ is the radius of the earth, $f$ the Coriolis parameter, $F$ is the friction force per unit mass and $\phi$ is the geopotential.

Since the wind speed varies much more rapidly in the vertical than in the horizontal, we shall neglect those contributions to $F$ which arise from the horizontal variations of the wind speed and write

where

$$
F \equiv g^{2} \frac{\partial}{\partial p}\left(K Q^{2} \frac{\partial u}{\partial p}\right)=A \frac{\partial}{\partial p}\left(p^{2} \frac{\partial u}{\partial p}\right)
$$

$$
A \equiv \frac{g^{2} K}{R^{2} \tilde{T}^{2}}=\mathrm{constant}
$$

and $g$ is the acceleration of gravity, $K$ is the coefficient of eddy viscosity (assumed constant), $\varrho$ is the density, $R$ is the gas constant and $\tilde{T}$ is some representative value of the temperature. The above formulation of the friction term is the same as that used by ROBERT (1966) in the time integration of his primitive equations model. In view of the quasi-geostrophic approximation the thermal wind equation

$$
\frac{\partial u}{\partial p}=\frac{R}{a f_{0} p} \frac{\partial T}{\partial \varphi}
$$

can be used to rewrite (2). In (3) $T$ is the temperature and $f_{0}$ is a representative value of $f$. Furthermore only the nondivergent part of the wind is retained on the left-hand side of (1), which means that the zonal average of the latter equation can be written in the form

where

$$
\frac{1}{a \cos ^{2} \varphi} \frac{\partial\left(M \cos ^{2} \varphi\right)}{\partial \varphi}=f_{0} \bar{v}_{z}+\frac{A R}{a f_{0}} \frac{\partial}{\partial p}\left(p \frac{\partial \bar{T}_{z}}{\partial \varphi}\right)
$$

$$
M(p, \varphi)={\overline{\left(u_{E} v_{E}\right)_{z}}}_{.}
$$


The subscript $z$ denotes the zonal average of the quantity, that is,

$$
()_{z}=\frac{1}{2 \pi} \int_{0}^{2 \pi}() d \lambda
$$

and the subscript $E$ denotes the eddy component, that is,

$$
()_{E}=()-()_{z} \text {. }
$$

Thus $M$ is the horizontal eddy transport of momentum, averaged with respect to time and longitude.

The thermodynamic equation, averaged with respect to time can be written as

$$
\frac{u}{a \cos \varphi} \frac{\partial \bar{T}}{\partial \hat{\lambda}}+\frac{v \overline{\partial T}}{a}-S \bar{\partial}=\frac{\bar{H}}{c_{p}}
$$

where $H$ is the diabatic heating per unit time and unit mass, and

$$
S=S(p)=\frac{R}{c_{p}} \frac{T_{S}}{p}-\frac{d T_{S}}{d p}
$$

is a measure of the static stability. $T_{S}$ is the temperature in some standard reference atmosphere and is a function of pressure only. It will be specified externally in the computations so that the model will not yield the area average of the temperature but rather the deviations from it.

If we take the zonal average of (6) and note that in the quasi-geostrophic formulation the temperature field is advected horizontally by the nondivergent part of the wind only, we find that the resulting equation can be written as

$$
\frac{1}{a \cos \varphi} \frac{\partial(N \cos \varphi)}{\partial \varphi}-S \bar{\omega}_{z}=\frac{\bar{H}}{c_{p}}
$$

where

$$
N(p, \varphi)=\overline{\left(v_{E} T_{E}\right)_{z}}
$$

is proportional to the meridional eddy transport of sensible heat, averaged with respect to longitude and time.

We shall consider the axisymmetric heating to be Newtonian in form so that

$$
\frac{\bar{H}_{z}}{\mathcal{c}_{p}}=q\left(T_{R}-\bar{T}_{z}\right)
$$

where $T_{R}(p, \varphi)$ is the equilibrium temperature toward which the diabatic processes are driving the atmosphere and $q$ is the heating or cooling coefficient. $T_{R}$ can be considered to be the temperature that (hypothetically) would be established in the atmosphere in the absence of the large-scale motion but in the presence of radiation and small-scale convention. CHARNEY [2] has discussed a heating mechanism of the 
form (10) for an atmosphere which was assumed to be isothermal in the vertical, transparent to solar radiation and 'gray' to terrestrial radiation. From his discussion we obtain a value of $0.34 \times 10^{-6} \mathrm{sec}^{-1}$ for $q$ whereas WIIN-NIELSEN, VERNEKAR and YANG [30] found a value of $0.4 \times 10^{-6} \mathrm{sec}^{-1}$ using the calculated value of the generation of eddy available potential energy from observations. Both of the above determinations of $q$ are based on a two-level representation of the atmosphere with only one temperature value in a vertical column and do not indicate how $q$ varies in the vertical. For lack of more adequate information a constant value of $q=0.4 \times 10^{-6} \mathrm{sec}^{-1}$ is used in the present study. The distribution of $T_{R}$, assumed known, will be discussed at a later stage.

To close our system of equations we introduce the continuity equation which, after being integrated with respect to time and longitude, can be written as

$$
\frac{1}{a \cos \varphi} \frac{\partial\left(\bar{v}_{z} \cos \varphi\right)}{\partial \varphi}+\frac{\partial \bar{\omega}_{z}}{\partial p}=0
$$

It is now possible to eliminate $\bar{v}_{z}$ and $\bar{\omega}_{z}$ from (4), (8) and (11) to obtain an equation in the single unknown $\bar{T}_{z}$. This is done by first substituting the expression for $\bar{v}_{z}$ from (4) into (11) to obtain

$$
\begin{aligned}
\frac{\partial \bar{\omega}_{z}}{\partial p}= & -\frac{1}{a^{2} f_{0} \cos \varphi} \frac{\partial}{\partial \varphi}\left[\frac{1}{\cos \varphi} \frac{\partial\left(M \cos ^{2} \varphi\right)}{\partial \varphi}\right] \\
& +\frac{A R}{a^{2} f_{0}^{2}} \frac{1}{\cos \varphi} \frac{\partial}{\partial \varphi}\left[\cos \varphi \frac{\partial}{\partial p}\left(p \frac{\partial \bar{T}_{z}}{\partial \varphi}\right)\right]
\end{aligned}
$$

We then integrate this equation from $p=0$ to an arbitrary $p$ using the boundary conditions

and

$$
\bar{\omega}_{z}=0 \text { at } p=0
$$

$$
p \frac{\partial \widetilde{T}_{z}}{\partial \varphi}=0 \text { at } p=0
$$

the latter of which is equivalent to saying that the frictional stress vanishes at $p=0$ (see (2) and (3)). We can finally eliminate $\bar{\omega}_{z}$ between the resulting equation and (8) to obtain, with the help of (10), the following differential equation for $\bar{T}_{z}$ :

where

$$
\frac{1}{\cos \varphi} \frac{\partial}{\partial \varphi}\left(\cos \varphi \frac{\partial \bar{T}_{z}}{\partial \varphi}\right)-\frac{q a^{2} f_{0}^{2}}{A S R p} \bar{T}_{z}=\frac{a^{2} f_{0}^{2}}{A S R p} G(p, \varphi)
$$

$$
\begin{aligned}
G(p, \varphi)= & -q T_{R}+\frac{1}{a \cos \varphi} \frac{\partial(N \cos \varphi)}{\partial \varphi} \\
& +\frac{S}{a^{2} f_{0} \cos \varphi} \frac{\partial}{\partial \varphi}\left[\frac{1}{\cos \varphi} \frac{\partial\left(I \cos ^{2} \varphi\right)}{\partial \varphi}\right]
\end{aligned}
$$


and

$$
I(p, \varphi)=\int_{0}^{p} M(p, \varphi) d p .
$$

We shall assume that $T_{R}, N, M$ and $S$ to be known so that the right-hand side of (12a) is known and hence the problem consists in solving (12a) subject to the appropriate boundary conditions. For lack of information about the Southern Hemisphere we shall require symmetry in $\widetilde{T}_{z}$ about the equator thus providing the boundary condition

$$
\frac{\partial \bar{T}_{z}}{\partial \varphi}=0 \text { at } \varphi=0 .
$$

The other boundary condition is provided by requiring that $\bar{T}_{z}$ be bounded at the poles.

The solution $T_{z}$ to (12a) can be obtained by expanding $G(p, \varphi)$ and $\bar{T}_{z}$ in terms of the even Legendre polynomials $P_{n}$, that is,

$$
\begin{aligned}
\bar{T}_{z}(p, \varphi) & =\sum_{n=0}^{N M} T_{n}(p) P_{n}(\sin \varphi) \\
G(p, \varphi) & =\sum_{n=0}^{N M} G_{n}(p) P_{n}(\sin \varphi)
\end{aligned}
$$

where $n$ is always even and

$$
G_{n}(p)=(2 n+1) \int_{0}^{2 \pi} G(p, \varphi) P_{n}(\sin \varphi) \cos \varphi d \varphi
$$

The coefficients $G_{n}$ in the expansion (13b) can therefore easily be determined since $G(p, \varphi)$ is known and the polynomials $P_{n}$ are readily obtained from the recursion formula

knowing that

$$
n P_{n}+(n-1) P_{n-2}-(2 n-1) \sin \varphi P_{n-1}=0
$$

$$
\begin{aligned}
& P_{0}=1 \\
& P_{1}=\sin \varphi .
\end{aligned}
$$

Substituting (13) into (12a) we find that the coefficients $T_{n}(p)$ are given by

$$
T_{n}(p)=-\frac{1}{q} \frac{m}{m+n(n+1)} \cdot G_{n}(p)
$$

where

$$
m=m(p)=\frac{q a^{2} f_{0}^{2}}{A S R p} .
$$

In the actual computations it was found sufficient to let $N M$, the maximum value of $n$, be 10 as the terms in the expansion (13a) decrease fairly rapidly in importance as 
$n$ increases. To some extent this is due to the fact that the frictional mechanism has introduced the factor $n(n+1)$ as part of the denominator on the right-hand side of (15). Clearly, the internal friction damps preferentially those components of $\bar{T}_{z}$ in (15) which have a large value of $n$, that is, a short meridional scale. It is also important to keep the maximum value of $n$ relatively small since $T_{R}$ and $I$ in (12b) are available at intervals of 10 degrees of latitude only.

\section{The data}

It is clear that to solve (12a) we need to know how the radiative equilibrium temperature $T_{R}$, the eddy heat transport $N$ and the eddy momentum integral $I$ vary with latitude at each pressure level where we want to obtain $\bar{T}_{z}$. Of the three required functions $N$ and $I$ are by far the better known. The eddy momentum transport values that were used are those computed by HoLOPAINEN [7] from the observed wind statistics for a period of about five years as published by CRUTCHER [4]. Holopainen's values for the fluxes due to the standing and transient eddies were added and a mean annual distribution of the sum was obtained as an average of the four seasons. The results appear in Table 1. All computations were then performed assuming that $M$ varies

Table 1

The eddy momentum transport based on Crutcher's (1959) data. Units: $\mathrm{m}^{2} \mathrm{sec}^{-2}$

\begin{tabular}{lrrrrrrr}
\hline Pressure (cb) & $15^{\circ} \mathrm{N}$ & $20^{\circ} \mathrm{N}$ & $30^{\circ} \mathrm{N}$ & $40^{\circ} \mathrm{N}$ & $50^{\circ} \mathrm{N}$ & $60^{\circ} \mathrm{N}$ & $70^{\circ} \mathrm{N}$ \\
\hline & & & & & & & \\
10 & 2.08 & 7.62 & 21.72 & 14.58 & 5.66 & -1.95 & -2.28 \\
20 & 11.10 & 19.12 & 42.36 & 41.8 & 20.06 & -0.72 & -3.50 \\
30 & 6.92 & 11.12 & 33.12 & 38.56 & 17.18 & -5.50 & -4.30 \\
50 & 3.00 & 4.30 & 12.52 & 15.00 & 6.86 & -1.20 & 0.65 \\
70 & 1.70 & 2.00 & 5.22 & 5.52 & 1.47 & -1.90 & -0.43 \\
85 & 0.27 & 0.95 & 3.82 & 1.35 & -0.40 & -2.20 & 0.57 \\
\hline
\end{tabular}

linearly in $\varphi$ and $p$ between data points and vanishes at $p=0$ and $100 \mathrm{cb}$ as well as at the equator and the pole.

The eddy heat transport data used in this study were computed by the method described by WIIN-NIELSEN, BROWN and DRAKE [27] on a daily basis for 1963 from data made available by the National Center for Atmospheric Research. The distribution of the mean annual eddy heat transport which was then obtained can be found in DEROME [5] for seven layers in the vertical and at intervals of 2.5 degrees of latitude. For the month of January the heat transport could not be computed for the layers 10 to $15 \mathrm{cb}, 15$ to $20 \mathrm{cb}$ and 85 to $100 \mathrm{cb}$ because of missing height data. For these layers the mean January eddy heat transport was computed using the assumption that the ratios of the missing heat transport data to those at the closest layer (also for 
January) where they were available was the same as the corresponding ratios for February.

At all pressure levels $N \cos \varphi$ and $\partial(N \cos \varphi) / \partial \varphi$ were taken to be zero at the equator and the values of $N \cos \varphi$ from $2.5^{\circ} \mathrm{N}$ to $15^{\circ} \mathrm{N}$ were computed by means of the polynomial

$$
N \cos \varphi=b_{1} \varphi^{3}+b_{2} \varphi^{5}
$$

in which $b_{1}$ and $b_{2}$ were evaluated by imposing the conditions that $N \cos \varphi$ and $\partial(N \cos \varphi) / \partial \varphi$ be continuous at $17.5^{\circ} \mathrm{N}$. An odd polynomial in $\varphi$ was chosen to extrapolate $N \cos \varphi$ in an effort to make each term on the right-hand side of (12b) symmetric about the equator.

Of the three terms on the right-hand side of (12b), the one containing the equilibrium temperature is the only one which cannot be obtained in a straightforward manner from the standard meteorological observations. In view of the uncertainty in the distribution of $T_{R}$ the computations were made with two different distrubutions. In distribution (a) the values of $T_{R}$ near the ground published by MiLankovitch [13] were used at $p=100 \mathrm{cb}$ while in distribution (b) the mean tropospheric radiative equilibrium temperatures obtained by SALTZMAN [19] (the average of his $\theta_{A 3}$ for winter and summer) were assumed to apply to the $60 \mathrm{cb}$ level and his surface temperature $\tau_{A 3}$ was used at $100 \mathrm{cb}$.

To obtain the vertical structure of $T_{R}$ it was assumed that in the absence of the large scale motion the troposphere and lower part of the stratosphere would tend to be in a state of radiative-convective equilibrium rather than in a state of pure radiative equilibrium since the latter would be gravitationally unstable (MANABE and STRICKLER [12]). It seems reasonable to expect that the temperature lapse rate in an atmosphere in convective-radiative equilibrium would be between the dry and moist adiabatic lapse rates and therefore not radically different from the average lapse rate in the atmosphere. This suggests that setting the lapse rate of $T_{R}$ equal to the latter would constitute a reasonable approximation.

WIIN-NIELSEN [25] has shown that the differential equation

$$
-S \equiv \frac{d T}{d p}-\frac{R}{c_{p}} \frac{T}{p}=-\frac{a}{p},
$$

where $a$ is a constant determined from observations, yields a solution for $T$ which closely approximates the average temperature in the lower atmosphere. It is found that the value $a=30^{\circ} \mathrm{K}$ gives a function $S(p)$ which is in close agreement with the observed distribution of $S$ published by GATES [6], when the average of his January and July distributions is considered. Given that $T=T_{0}$ at $p=p_{0}$, the solution of (16) is

$$
T=T_{0}\left(\frac{p}{p_{0}}\right)^{R / c_{p}}+\frac{c_{p} a}{R}\left[1-\left(\frac{p}{p_{0}}\right)^{R / c_{p}}\right] .
$$

This empirical formula, with $a=30^{\circ} \mathrm{K}$ and $p_{0}=100 \mathrm{cb}$ for case (a) and $p_{0}=60 \mathrm{cb}$ 
for case (b), was used to compute $T_{R}$ at $p=20,30,50,70$ and $85 \mathrm{cb}$. The values of $T_{R}$ at 10 and $15 \mathrm{cb}$ were assumed to be the same as those at $20 \mathrm{~cm}$ to simulate the lower stratosphere. Of distributions (a) and (b) for $T_{R}$, which appear as the dashed lines in figures 4 and 5 (as departures from the area mean values), the first yields results for the verifiable variables, such as $\bar{T}_{z}$ and $\bar{u}_{z}$, which agree better with observations than those of the second distribution. This leads us to believe that the less easily verifiable quantities, such as $\bar{\omega}_{z}$ obtained from case (a) are probably closer to reality than those of case (b) and will be given correspondingly more attention.

The constant $A$ appearing in (12a) was evaluated (see (2)) using $\tilde{T}=250^{\circ} \mathrm{K}$ and $K=45 \mathrm{~m}^{2} \mathrm{sec}^{-1}$. With a representative density of $0.5 \times 10^{-3} \mathrm{t} \mathrm{m}^{-3}$ this value of $K$ corresponds to a value of $\mu=\varrho K=2.25 \times 10^{-2} \mathrm{t} \mathrm{m}^{-1} \mathrm{sec}^{-1}$, which is equal to PALMÉN's [15] estimate. The static stability parameter $S$ in (12) was evaluated as a function of pressure from GATES' [6] observational study of the static stability by averaging his values for $S$ for January and July.

\section{The temperature distribution}

The differential equation (12a) was solved for $\widetilde{T}_{z}$ by the method described above. The equation is linear so that the contributions to the solution $\bar{T}_{z}$ by the three terms defining $G(p, \varphi)$ are additive. In fact, the sum of their contributions gives the complete solution since the homogeneous part of (12a) has no solution satisfying the boundary conditions.

To determine the role that the eddies play in maintaining the axisymmetric temperature field we shall compute $\bar{T}_{z}$ firstly under the conditions $T_{R} \equiv M \equiv 0, N \equiv 0$, thus obtaining the effect of the eddy heat transport on $\bar{T}_{z}$, then under the conditions $T_{R} \equiv N \equiv 0, M \not \equiv 0$, thereby obtaining the influence of the eddy momentum transport, and thirdly under the condition $T_{R} \equiv 0, N \neq 0, M \not \equiv 0$, to obtain the total contribution by the eddies to $\bar{T}_{z}$. We shall then present the case $T_{R} \not \equiv 0, M \equiv N \equiv 0$ as well as the complete solution of (12).

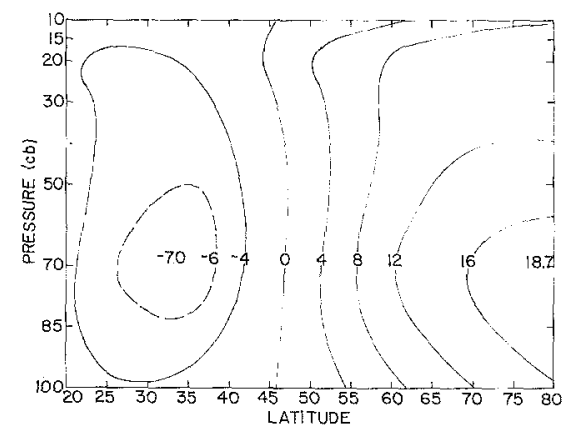

Figure 1

The field of $\bar{T}_{z}$, in degrees Kelvin, as forced by the horizontal eddy heat transport 
The contribution to $\bar{T}_{z}$ by the horizontal eddy heat transport $N$ appears in the form of a meridional-vertical cross section in figure 1. A positive (negative) value of temperature at a given point indicates that the eddy heat transport acts to raise (lower) the temperature at that point. The main features of the results, namely, the cooling and warming of the low and high latitudes, respectively, follow directly from the observed fact that on the average the eddies transport heat northward with a maximum in the middle latitudes. We note, in particular, that the computed effect of the eddy heat transport on the temperature field is strongest near $70 \mathrm{cb}$ and at high latitudes.

Some computations were also made using a coefficient of diffusion $K=90 \mathrm{~m}^{2} \mathrm{sec}^{-1}$ rather than $45 \mathrm{~m}^{2} \mathrm{sec}^{-1}$. The results, which can be seen in DeROME [5], are qualitatively similar to those of figure 1 . In particular, the positions of the minimum and maximum temperatures, as well as the zero line, are virtually the same. The main difference can be found in the fact that the minimum and maximum temperatures have values of -4.7 and $14.6^{\circ} \mathrm{C}$ rather than -7.0 and $18.7^{\circ} \mathrm{C}$.

In view of (10) we have, for the case $T_{R} \equiv 0$

$$
\bar{H}_{z}=-q^{*} \bar{T}_{z}
$$

where $q^{*}=c_{p} q$. We can therefore obtain the diabatic heating rate $\bar{H}_{z}$ for the present case by multiplying $\bar{T}_{z}$ in figure 1 by $-q^{*}=-0.4 \times 10^{-3} \mathrm{kj} \mathrm{t}^{-1} \mathrm{deg}^{-1} \mathrm{sec}^{-1}$. It is clear that in this case the low and high latitudes are regions of diabatic heating and cooling, respectively.

The contribution to $\bar{T}_{z}$ from the eddy momentum transport alone appears in figure 2. This is the temperature field which produces just enough diabatic heating or

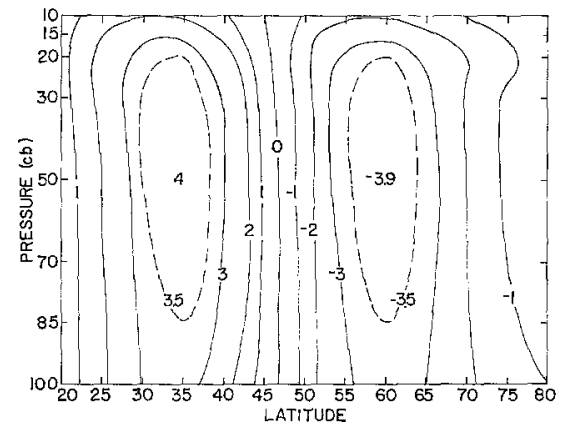

Figure 2

The field of $\bar{T}_{z}$, in degrees Kelvin, as forced by the horizontal eddy momentum transport

cooling to balance the adiabatic cooling or heating by the mean meridional circulation which, in turn, is forced by the eddy momentum transport. From (18) and figure 2 we see that there is some diabatic cooling and downward motion in the region between $20^{\circ} \mathrm{N}$ and about $47^{\circ} \mathrm{N}$ whereas the region to the north is one of diabatic heating and upward motion. 
Figures 1 and 2 indicate that the eddy momentum transport has its maximum effect on the temperature field at a higher level than does the eddy heat transport. We note also that the effect of the eddy momentum transport on $\bar{T}_{z}$ is rather small near the pole in contrast to that of the eddy heat transport which is quite large. Perhaps the most striking feature about figures 1 and 2 is the tendency that the heat and momentum transports have to oppose each other in the way they affect $\widetilde{T}_{z}$. The sum of the two figures, representing the total effect of the eddies on the temperature field appears in figure 3 . We see that the eddies cool the low latitudes and warm the high latitudes with the maximum effects being found near $70 \mathrm{cb}$.

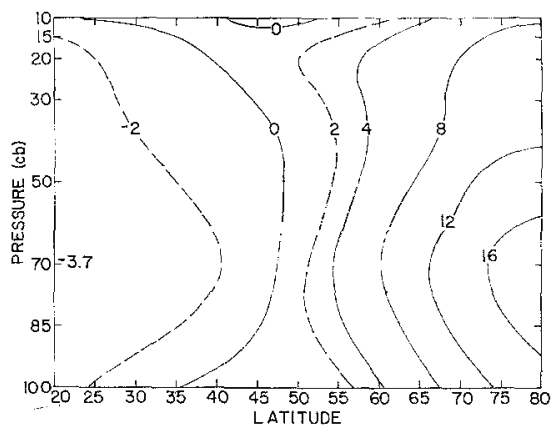

Figure 3

The field of $\bar{T}_{z}$, in degrees Kelvin, as forced by the combined action of the eddy heat and momentum transports
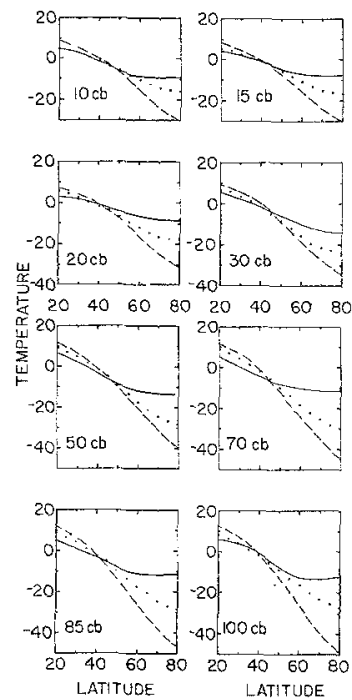

Figure 4

The dotted curves give the solution $\bar{T}_{z}$ as forced by distribution (a) for $T_{R}$ (dashed curves). The solid curves show the solution $\widetilde{T}_{z}$ as forced by the combined action of $T_{R}$ together with the eddy heat and momentum transports. The temperatures are in degrees Kelvin and are deviations from their respec- 
The contribution to $\bar{T}_{z}$ by the function $T_{R}$ (distribution (a)) appears as the dotted curves in figure 4 while $T_{R}$ itself is given by the dashed curves. The difference between the dashed and dotted curves is due to the presence of friction. We note that in the absence of the eddies the vertical motion is given by

$$
\bar{\omega}_{z}=\frac{q}{S}\left(\bar{T}_{z}-T_{R}\right)
$$

so that for the present case we have a one cell circulation with upward motion in the low latitudes (where there is diabatic heating since $\bar{T}_{z}<T_{R}$ ) and downward motion in the high latitudes (where there is diabatic cooling since $\bar{T}_{z}>T_{R}$ ).

The complete solution to (12a), determined as the sum of the various contributions discussed so far, appears as the solid curves in figure 4 . Whereas the difference between the dashed and dotted curves is due to the one-cell mean meridional criculation present in the absence of the eddies, the difference between the solid and dotted curves results from the presence of the eddies, whose influence on the temperature field has already been discussed. It is clear from a comparison of the solid and dashed curves that at all levels shown the large scale eddies and friction mechanism force the temperature difference between $20^{\circ} \mathrm{N}$ and $80^{\circ} \mathrm{N}$ to be appreciably smaller than in the thermal equilibrium condition. For example, at $100 \mathrm{cb}$ the computed temperature difference is $18^{\circ} \mathrm{K}$, as compared to $61^{\circ} \mathrm{K}$ in the equilibrium temperature.

The average observed temperature for the one year period starting in February 1963 appears in figure 5. This figure is presented in a different format from that of figure 4 because the lack of data south of $25^{\circ} \mathrm{N}$ made it impossible to compute and subtract from the data the hemispheric mean temperature. We see that the agreement between the observed and computed meridional temperature variations is generally

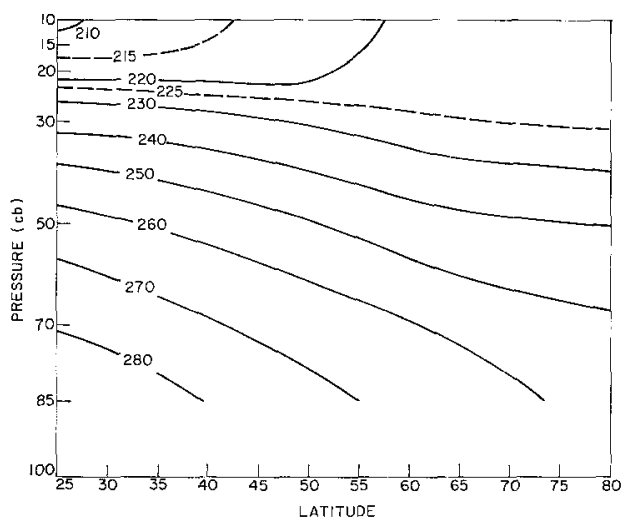

Figure 5

The observed axisymmetric temperature field average over the one year period beginning in February 1963, in degrees Kelvin 
quite good in the troposphere. For example, at $50 \mathrm{cb}$ a computed temperature difference of $18^{\circ} \mathrm{C}$ is obtained while a difference of about $23^{\circ} \mathrm{C}$ is observed. Furthermore, the computed meridional temperature gradient in the mid-troposphere is seen to be larger in the middle latitudes than further north and south, in agreement with observations. In the lower stratosphere, on the other hand, the computations fail to reproduce the observed increase in temperature from south to north. Part of this discrepancy may be due to an improper specification of the equilibrium temperature in this region, but on the other hand it is also possible that one or more of our modelling assumptions (such as taking the static stability to be independent of latitude) is less valid in the lower stratosphere than in the troposphere (see MANABE and HunT [10]).

The results obtained with distribution (b) for $T_{R}$ appear in figure 6 . We find that

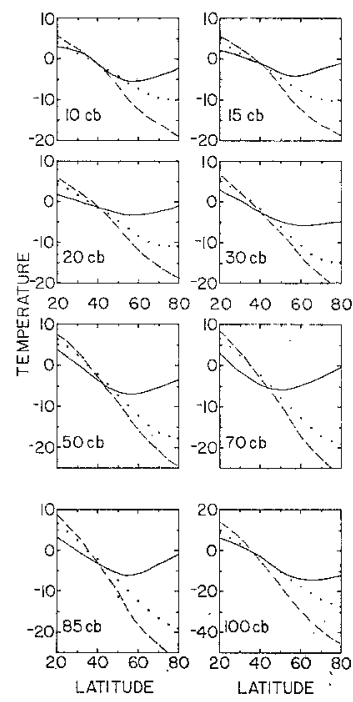

Figure 6

Same as figure 4 but with distribution (b) for $T_{R}$. Note the difference in the temperature scales between figures 4 and 6

in general the values of $T_{R}$ in northern latitudes are not sufficiently low to counteract the effects of the large scale eddies and friction with the result that the minimum temperatures (solid lines) occur too far south. It is interesting to note that at $100 \mathrm{cb}$ the values of $T_{R}$ (from SALTZMAN [19]) are practically identical with those of figure 4 (from Milankovitch [13]). This means that if we had used only Saltzman [19] surface equilibrium temperature distribution and derived from it the vertical profile of $T_{R}$ as we did with MiLANKOVITCH's [13] data (distribution (a)), the results would have been nearly identical with those of figure 4 .

In the next section we shall present the mean meridional circulation which is in equilibrium with the temperature distribution of figure 4 . 


\section{The mean meridional circulation}

Once $\bar{T}_{z}$ is known the vertical velocity $\bar{\omega}_{z}$ can easily be obtained from (8) and (10). Figure 7 shows the result of the computations for the case where the heat and momentum transports are included and $T_{R}$ is as given in figure 4 . The direction of the

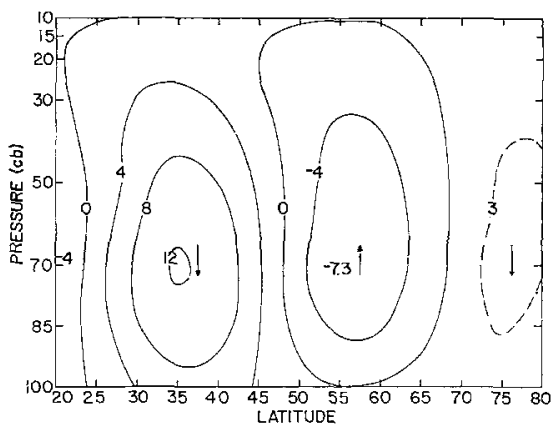

Figure 7

The field of $\omega_{z}$, in units of $10^{-6} \mathrm{cb} \mathrm{sec}^{-1}$, resulting from the diabatic heating and the horizontal eddy transports of heat and momentum

arrows indicates whether the air is moving toward a higher or lower pressure. It may be mentioned for convenience here that at $70 \mathrm{cb}$ a value of $\bar{\omega}_{z}=10 \times 10^{-6} \mathrm{cb} \mathrm{sec}^{-1}$ corresponds to a vertical velocity of about $1 \mathrm{~mm} \mathrm{sec}^{-1}$. We find some upward motion from $20^{\circ} \mathrm{N}$ to about $24^{\circ} \mathrm{N}$, downward motion from $24^{\circ} \mathrm{N}$ to about $47^{\circ} \mathrm{N}$ changing into upward motion to about $67^{\circ} \mathrm{N}$ and finally some downward motion from $67^{\circ} \mathrm{N}$ to $80^{\circ} \mathrm{N}$. This distribution is in good agreement with the results obtained by VERNEKAR [23] in his extensive study of the mean meridional criculation.

In view of (11), $\bar{\omega}_{z}$ and $\bar{v}_{z}$ can be written in terms of a stream function $\psi(p, \varphi)$ as

$$
\begin{gathered}
\bar{\omega}_{z}=-\frac{1}{\cos \varphi} \frac{\partial \psi}{\partial \varphi} \\
\bar{v}_{z}=\frac{a}{\cos \varphi} \frac{\partial \psi}{\partial p} .
\end{gathered}
$$

The boundary conditions on $\psi$ are obtained by first noting that the symmetry of $\bar{T}_{z}$ and $M$ about the equator implies, through (4), that $\bar{v}_{z}(p, o)=0$ so that

$$
\psi(p, o)=\text { constant } .
$$

Similarly the boundary condition $\bar{\omega}_{z}(o, \varphi)=0$ implies that

$$
\psi(o, \varphi)=\text { constant }
$$

and the condition that $\bar{v}_{z}(p, \pi / \alpha)$ be finite implies, through (19b), that

$$
\psi(p, \pi / 2)=\text { constant } .
$$


Finally the continuity of $\psi$ requires that the constants in $(20 \mathrm{a}, \mathrm{b}, \mathrm{c})$ be equal to each other. For convenience we set the constant equal to zero.

The integral of (19a) which satisfies these boundary conditions is simply

$$
\psi(p, \varphi)=-\int_{0}^{\varphi} \bar{\omega}_{z} \cos \varphi d \varphi .
$$

The stream function corresponding to the $\bar{\omega}_{z}$ field of figure 7 appears in figure 8 . The arrows indicate schematically the direction of flow. If we compare this cross section

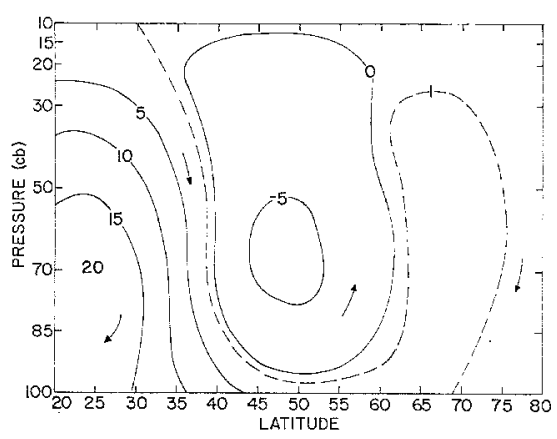

Figure 8

The stream function $\psi$, in units of $10^{-7} \mathrm{cb} \mathrm{sec}^{-1}$, corresponding to the $\omega_{z}$ field of figure 7

with those obtained by STARR et al. [21] using observed wind data (analysed by six different procedures) for the five year period starting in May 1958 we find a number of similarities. In both studies the center of the Ferrel cell is located in the vicinity of $45^{\circ} \mathrm{N}$ and at about $60 \mathrm{cb}$ and the cell itself occupies the entire depth of the troposphere. Similarly in both studies the polar cell is centered near $65^{\circ} \mathrm{N}$. As for the Hadley circulation, the comparison between our results and those of STARR et al. is made practically impossible by the fact that the results of the latter are rather sensitive to the analysis procedures that were used. In the present study we obtain, for the region south of about $30^{\circ} \mathrm{N}$, a northerly flow from $100 \mathrm{cb}$ to about $70 \mathrm{cb}$ and a southerly flow aloft.

\section{The mean zonal wind}

To obtain the speed of the zonal current we integrate the thermal wind equation (3) so that $\bar{u}_{z}$ takes the form

$$
\bar{u}_{z}(p, \varphi)=\bar{u}_{z}\left(p_{g}, \varphi\right)+\frac{R}{a f_{0}} \frac{\partial}{\partial \varphi} \int_{p_{g}}^{p} \frac{\bar{T}_{z}}{p} d p
$$

where $p_{g}$ is the pressure at the ground. The zonal wind at the ground is in turn evalua12 PAGEOPH 95 (1972/III) 
ted by first writing the friction term in (2) as

$$
F=g \frac{\partial \tau}{\partial p}
$$

where $\tau$ is the zonal component of eddy stress, so that (4) becomes

$$
\frac{1}{a \cos ^{2} \varphi} \frac{\partial\left(M \cos ^{2} \varphi\right)}{\partial \varphi}=f_{0} \bar{v}_{z}+g \frac{\partial \bar{\tau}_{z}}{\partial p} .
$$

If we neglect the presence of the continental elevations so that $\bar{\omega}_{z}=0$ at the earth's surface, we find from the continuity equation that

$$
\int_{p_{g}}^{0} \bar{v}_{z} d p=0
$$

so that integrating (24) from $p=0$, where $\bar{\tau}_{z}=0$ to $p=p_{g}$ we obtain

$$
\bar{\tau}_{z, g}(\varphi)=\frac{1}{g a \cos ^{2} \varphi} \frac{\partial\left(I_{\mathrm{g}} \cos ^{2} \varphi\right)}{\partial \varphi}
$$

where the subscript $g$ identifies the value of the variable at the ground and $I$ is as defined by (12c). If we now assume that the stress at the ground is given by

$$
\bar{\tau}_{z, g}(\varphi)=-C_{d} V \varrho_{g} u_{z}\left(p_{g}, \varphi\right)
$$

where $C_{d}$ is the drag coefficient, $\varrho_{g}$ the density at the ground and $V$ a characteristic wind speed, we obtain the following equation for the wind speed at the ground:

$$
\bar{u}_{z}\left(p_{g}, \varphi\right)=-\frac{1}{g a C_{d} V \varrho_{g} \cos ^{2} \varphi} \frac{\partial\left(I_{g} \cos ^{2} \varphi\right)}{\partial \varphi} .
$$

We shall follow Phiclips [16] and use the following values for the parameters: $C_{d}=3 \times 10^{-3}, \varrho_{g}=10^{-3} \mathrm{t} \mathrm{m}^{-3}, V=10 \mathrm{~m} \mathrm{sec}^{-1}$.

The contributions to the complete solution $\bar{u}_{z}(p, \varphi)$ by the eddy heat transport, the eddy momentum transport and the equilibrium temperature distribution are additive so that it is a simple matter to investigate the importance of the eddies in determining the speed of the zonal flow. For this purpose we have computed the distribution of under the following special conditions:

(a) $N \neq 0, M \equiv T_{R} \equiv 0$

(b) $M \neq \equiv, N \equiv T_{R} \equiv 0$

(c) $N \neq 0, M \not \equiv 0, T_{R} \equiv 0$

(d) $N \equiv M \equiv 0, T_{R} \not \equiv 0$

(e) $N \neq 0, M \not \equiv 0, T_{R} \not \equiv 0$.

Those are the same cases for which $\bar{T}_{z}(p, \varphi)$ has been computed previously.

The solution for case (a), giving the contribution by the eddy heat transport to 
the complete solution for $\bar{u}_{z}(p, \varphi)$ appears in figure 9. This zonal wind distribution is in geostrophic equilibrium with the temperature field of figure 1 , with zero wind speed at the earth's surface since in the absence of the eddy momentum transport $\bar{u}_{z}=0$ at $p=p_{g}$. The results indicate that south of about $30^{\circ} \mathrm{N}$ the eddy heat transport tends to produce weak westerlies while further north the tendency is to produce easterlies which increase in speed with height, reaching a maximum value of $38 \mathrm{~m} \mathrm{sec}^{-1}$ at $10 \mathrm{cb}$.

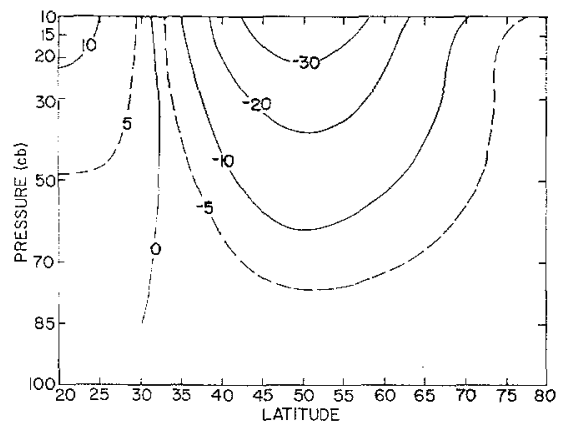

Figure 9

The contribution by the horizontal eddy heat transport to the zonal wind $\bar{u}_{z}$, in $\mathrm{m} \mathrm{sec}^{-1}$

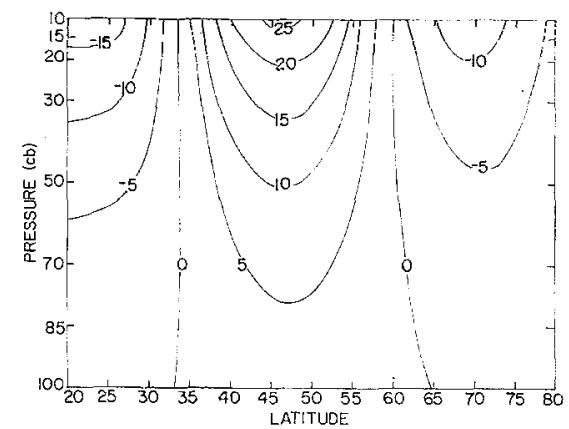

Figure 10

The contribution by the horizontal eddy momentum transport to the zonal wind $\vec{u}_{z}$, in $\mathrm{m} \mathrm{sec}^{-1}$

The contribution by the eddy momentum transport to the mean zonal wind can be found in figure 10. This distribution of $\tilde{u}_{z}$ is the one obtained for case (b) and is in geostrophic equilibrium with the temperature field of figure 2 . It is seen that the eddies transport momentum in such a way as to produce westerlies from about $34^{\circ} \mathrm{N}$ to about $60^{\circ} \mathrm{N}$ and easterlies south of $34^{\circ} \mathrm{N}$ and north of $60^{\circ} \mathrm{N}$. In each of the three regions the maximum influence of the eddy momentum transport is found at $10 \mathrm{cb}$, the highest level for which computations were made. This result deserves some comments in view of the fact that the eddy momentum transport (see Table 1) does not have its relative maxima at $10 \mathrm{cb}$ but rather at 20 or $30 \mathrm{cb}$ depending on the latitude. Further- 
more, observational studies such as the one by WIIN-NieLSEN [26] have shown that the rate of kinetic energy conversion between the eddies and the zonal flow is a maximum near $20 \mathrm{cb}$. To understand the results of the present study we must realize that while the eddies are more effective at transporting and transferring their momentum to the zonal flow at $20 \mathrm{cb}$ than at $10 \mathrm{cb}$, they are also quite effective at creating a mean meridional criculation. The computations of the stream function show that the eddy momentum transport results in a two-cell mean meridional circulation with, in particular, the maximum northerly flow in the middle latitudes occurring between 20 and $30 \mathrm{cb}$. Through the action of the Coriolis force this circulation tends to create easterlies near the middle latitude tropopause in opposition to the other effect through which the eddies tend to create a source of eastward momentum for the zonal current. A discussion of these two effects as computed from observations can be found in WIINNielsen and VerneKar [29] but since their mean meridional circulation was produced by both the eddy heat and momentum transports their results are not directly comparable to ours. We should also note that the vertical diffusion of momentum, which tends to smooth the vertical profile of $\bar{u}_{z}$, does not play a leading role in the above argument since some computations done without diffusion yielded results which agree qualitatively with those of figure 10 .

If we add the values of $\bar{u}_{z}$ from figures 9 and 10 we obtain the solution of case (c) as shown in figure 11 . The results indicate that the combined effect of the heat and

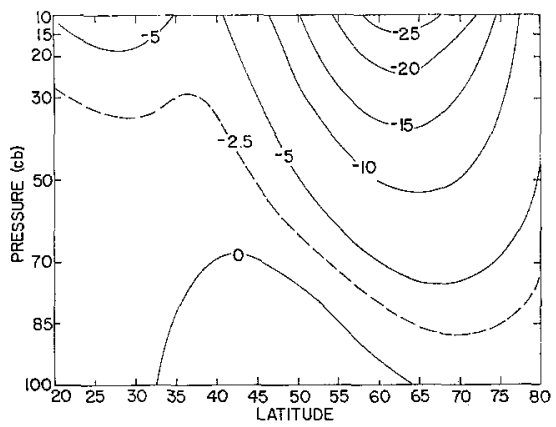

Figure 11

The sum of the contributions shown in figures 9 and 10

momentum transports on the zonal current is to produce weak westerlies (maximum value of $2.3 \mathrm{~m} \mathrm{sec}^{-1}$ ) in a relatively shallow layer in the middle latitudes and easterlies elsewhere. The easterlies are relatively weak in the southern latitudes but reach speeds of up to $28 \mathrm{~m} \mathrm{sec}^{-1}$ in the upper level near $65^{\circ} \mathrm{N}$.

The solution for $\bar{u}_{z}$ determined by distribution (a) for $T_{R}$ and the influence of internal friction appears in figure 12. This distribution of $\tilde{u}_{z}$ is in geostrophic equilibrium with the temperature values given by the dotted curves in figure 4 . In the absence of the eddies the zonal flow would then consist of a broad westerly current in which 


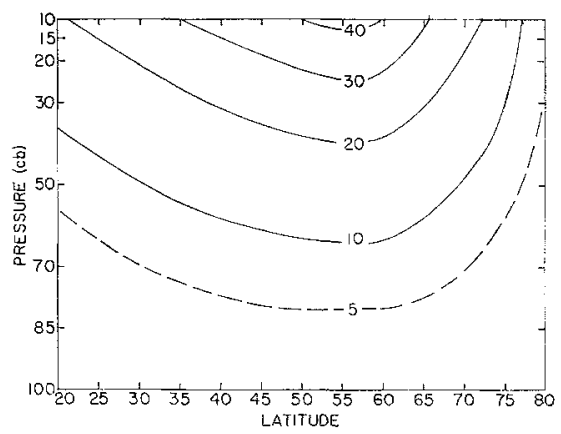

Figure 12

The contribution by the function $T_{R}$ (distribution (a)) to the zonal wind $\bar{u}_{2}$ in $\mathrm{m} \mathrm{sec}^{-1}$

the wind speed increases monotonically with height. We should stress, on the other hand, that in view of the way $T_{R}$ was obtained the values of $\bar{T}_{z}$ given by the dotted curves of figure 4 are probably less reliable at the upper levels than at the lower levels and hence the vertical wind shear at the upper levels in figure 12 should be considered with some caution. Nevertheless it is instructive to investigate how far we can go in trying to reproduce the observed zonal wind by adding the contributions shown in figures 11 and 12.

The result of adding the contributions to $\bar{u}_{z}$ by the eddies and the function $T_{R}$ (distribution (a)) appears in figure 13 and represents the complete solution for $\bar{u}_{z}$. Figure 14 presents the corresponding results for the case where distribution (b) for $T_{R}$ is used. Figure 15 shows the observed geostrophic zonal wind averaged over the period February 1963 through January 1964. In the middle latitude troposhere the computed zonal wind of figure 13 agrees quite well with observations. In both, for example, the wind has a jet structure whose axis slopes to the south with height in the lower troposphere and becomes nearly vertical in the upper levels. The main deficiency to be noticed in the computed wind field is the absence of the vertical wind shear

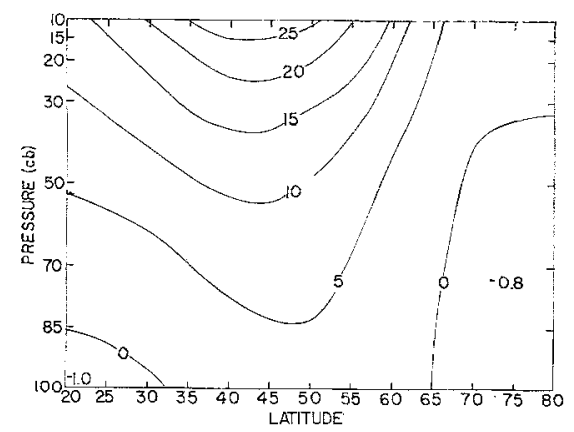

Figure 13

The total solution for the zonal wind $\bar{u}_{z}$ in $\mathrm{m} \mathrm{sec}^{-1}$, as forced the eddy heat and momentum transports and by distribution (a) for $T_{R}$ 


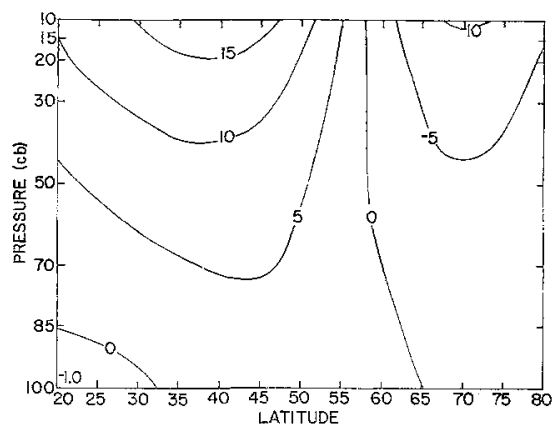

Figure 14

Same as figure 13 but with distribution (b) for $T_{R}$

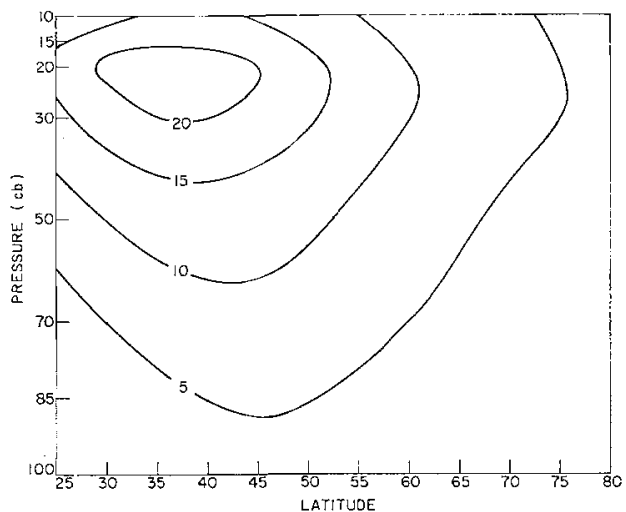

Figure 15

The distribution of the observed geostrophic wind, in $\mathrm{m} \mathrm{sec}^{-1}$, averaged over the one year period beginning in February 1963

reversal in the lower stratosphere. This discrepancy, of course, was to be expected from the computed temperature field discussed earlier. It is worth noting that a similar deficiency in the zonal wind field was also present in the general circulation experiment of SMAGORINSKY et al. [20] and, to a lesser extent, in that of MANABE et al. [11].

When distribution (b) for $T_{R}$ is used, the mean zonal wind (figure 14) does not agree as well with observations. It appears that the meridional gradient of $T_{R}$ is somewhat too small, leading to westerlies that are weaker than the observed ones in the middle latitude and to relatively strong easterlies in the northern latitudes, where weak westerlies are observed. We are led to the conclusion, then, that distribution (a) for $T_{R}$ is more nearly compatible with our model than distribution (b).

Finally, some computations were made to determine how the results would be 
affected by a change in the coefficient of eddy viscosity, $K$. It was found, in particular, that doubling the value of $K$ reduces the maximum wind speed of $29.1 \mathrm{~m} \mathrm{sec}^{-1}$ in figure 13 to $22.9 \mathrm{~m} \mathrm{sec}^{-1}$ and restricts the polar easterlies to the layer below about $60 \mathrm{cb}$. Setting the coefficient of eddy viscosity equal to zero, on the other hand, results in a maximum wind speed of $59.2 \mathrm{~m} \mathrm{sec}^{-5}$ at $10 \mathrm{cb}$ together with a band of polar easterlies at all levels, reaching $19.6 \mathrm{~m} \mathrm{sec}^{-1}$ at $10 \mathrm{cb}$. Similarly, the maximum wind speed of $19.6 \mathrm{~m} \mathrm{sec}^{-1}$ in figure 14 increases to $46.4 \mathrm{~m} \mathrm{sec}^{-1}$ when the internal diffusion of momentum is removed, and the maximum easterly speed of $-10.6 \mathrm{~m} \mathrm{sec}^{-1}$ reaches $-42.5 \mathrm{~m} \mathrm{sec}^{-1}$. It appears, then, that the friction mechanism plays a necessary role in the above formulation but that the results are not excessively sensitive to a change in $K$ by even a factor of two.

\section{Summary and concluding remarks}

By using a quasi-geostrophic formulation of the meteorological equations with internal friction and assuming the axisymmetric diabatic heating to be Newtonian it has been possible to show how the axisymmetric temperature field is affected by the eddy heat and momentum transports as well as by the assumed distribution of the convective-radiative equilibrium temperature. The results indicate that the eddy heat and momentum transports tend to oppose each other in the way they affect the middle latitude temperature field. The combined effect of the two mechanisms is to create a temperature difference between $16^{\circ} \mathrm{C}$ in the high latitudes and about $-4^{\circ} \mathrm{C}$ in the low latitudes.

From the solution obtained for the temperature field it has also been possible to determine the velocity field. The mean meridional circulation thus obtained shows reasonable agreement with those of previous investigations. A study was also made of the role played by the eddies in determining the geostrophic zonal wind. It was found that the combined effect of the eddy heat and momentum transports is to produce weak westerlies near the ground in the middle latitudes and easterlies elsewhere, the latter reaching speeds of up to $28 \mathrm{~m} \mathrm{sec}^{-1}$ at the highest level $(10 \mathrm{cb})$ at $60^{\circ} \mathrm{N}$. The final solution for the zonal wind was then obtained by adding the wind distribution produced by the eddies to the one that would exist if the atmosphere were in a state of convective-radiative equilibrium. The resulting zonal wind was found to be in good agreement with observations in the middle latitude troposphere while in the lower stratosphere the model has failed to reproduce the observed decrease of westerly wind speed with height.

The approach in this study has been to rely on observations for the evaluation of the eddy heat and momentum transports. This procedure is useful in that it can serve as a guide in parameterizing the influence of the large-scale eddies on the zonally averaged flow, a problem which has already been treated in the literature (e.g. Adem [1], Williams and Davies [31], Saltzman [19] and Clapp [3]) but which still requires further attention. 


\section{Acknowledgements}

The authors would like to thank Mr. RENÉ BoISVERT who wrote most of the computer programs used in this study and Mr. Yvon BouRASSA who drafted the figures. The research was supported in part by N.S.F. Grants GP-2561 and GA-841.

\section{REFERENCES}

[1] J. ADEM, On the theory of the general circulation of the atmosphere, Tellus 14 (1962), 102-115.

[2] J. Charney, On the theory of the general circulation of the atmosphere, The Atmosphere and the Sea in Motion, B. Bolin, ed. (The Rockefeller Institute Press, New York, 1959), pp. 178-193.

[3] P. F. ClAPP, Parameterization of macroscale transient heat transport for use in a mean-motion model of the general circulation, J. Appl. Meteorol. 9 (1970), 554-563.

[4] H. L. CRUTCHER, Upper wind statistics charts of the northern hemisphere. Office of the Chief of the U.S. Naval Operations, Washington, D.C. (1959).

[5] J. F. Derome, The maintenance of the time-averaged state of the atmosphere, Ph. D. Thesis (available as Tech. Report ORA 08759-2T), Department of Meteorology and Oceanography, The University of Michigan, Ann Arbor (1968).

[6] L. W. GATES, Static stability measures in the atmosphere, J. Meteorol. 18 (1961), 526-533.

[7] E. O. HolopaINEN, On the mean meridional circulation and the flux of angular momentum over the northern hemisphere, Tellus 19 (1967), 1-13.

[8] H. G. Houghton, On the annual heat balance of the northern hemisphere, J. Meteorol. 11 (1954), $1-9$.

[9] H. L. Kuo, Forced and free meridional circulations in the atmosphere, J. Meteorol. 13 (1956), 561-568.

[10] S. MANABE and B.G. HUNT, Experiments with a stratospheric general circulation model: I. Radiative and dynamic aspects, Mon. Weather Rev. 96 (1968), 477-502.

[11] S. Manabe, J. Smagorinsky, J. L. Holloway, and H. M. Stone, Simulated climatology of a general circulation model with a hydrologic cycle. III. Effects of increased horizontal computational resolution, Mon. Weather Rev, 98 (1970), 175-212.

[12] S. MANABE and R. F. STRICKLER, Thermal equilibrium of the atmosphere with a convective adjustment, J. Atmos, Sci. 12 (1964), 361-385.

[13] M. MILANKOVITCH, Mathematische Klimalehre und astronomische Theorie der Klimaschwankungen, Handbuch der Klimatologie, W. Köppen and R. Geiger, eds., Vol. 1, A (1930), 1-176.

[14] Y. MINTZ, Very long-term global integration of the primitive equations of atmospheric motion, in Proceedings of WMO-IUGG Symposium on Research and Development Aspects of Long Range Forecasting, Boulder, Colorado, WMO Tech. note, No. 66 (1964), 141-167.

[15] E. PALMÉN, On the mean meridional circulation in low latitudes of the northern atmosphere in winter and the associated meridional and vertical fux of angular momentum, Societas Scientiarium Fennica, Commentationes Physico-Mathematicae, Finska Vetenskaps-Societeten, Helsinki, 17 (1955), 1-33.

[16] N. A. PHILlIPS, The general circulation of the atmosphere: A numerical experiment, Q. J. Roy. Meteorol. Soc. 82 (1956), 123-164.

[17] E. R. ReITER, Atmospheric transport processes, Part 1: Energy transfers and transformations, Report TID-24868, USAEC Division of Technical Information, Oak Ridge, Tenn. (1969), 253 pp.

[18] A. J. ROBERT, The integration of a low order spectral form of the primitive meteorological equations, J. Meteorol. Soc. Japan, Series II, 44 (1966), 237-245.

[19] B. SAltzman, Steady state solutions for axially-symmetric climatic variables, Pure Appl. Geophys. 69 (1968), 237-259.

[20] J. SMAGORINSKY, S. MANABE and J. LeITH $J_{\mathrm{R}}$., Numerical results from a nine-level general circulation model of the atmosphere, Mon. Weather Rev. 93 (1965), 727-768.

[21] V. P. STARR, J. P. PEIXoTo and N. E. GAUT, Momentum and zonal kinetic energy balance of the atmosphere from five years of hemispheric data, Tellus 22 (1970), 251-274. 
[22] V.P. StARR and R.M. WhITE, A hemispheric study of the atmospheric angular-momentum balance, Q.J. Roy. Meteorol. Soc. 77 (1951), 215-225.

[23] A. D. Vernekar, On mean meridional circulations in the atmosphere, Mon. Weather Rev. 95 (1967), 705-721.

[24] W. M. WASHINGTON and A. KASAHARA, A January simulation experiment with a two-layer version of the NCAR global circulation model, Mon. Weather Rev. 98 (1970), 559-580.

[25] A. WIIN-NIELSEN, On barotropic and baroclinic models with special emphasis on ultra-long waves, Mon. Weather Rev. 87 (1959), 171-183.

[26] A. WIIN-NreLSEN, On the annual variation and spectral distribution of atmospheric energy, Tellus 19 (1967), 540-559.

[27] A. WIIN-NIELSEN, J. A. BRoWn and M. DRAKE, On atmospheric energy conversions between the zonal flow and the eddies, Tellus 15 (1963), 261-279.

[28] A. WiIn-Nielsen, J. A. Brown and M. Drake, Further studies of energy exchange between the zonal flow and the eddies, Tellus 16 (1964), 168-180.

[29] A. WIIN-NIELSEN and A. D. VeRneKAR, On the influence of the mean meridional circulation on the zonal flow, Mon. Weather Rev. 95 (1967), 723-732.

[30] A. Winn-Nielsen, A. D. VerneKaR and C. H. YANG, On the development of baroclinic waves influenced by friction and heating, Pure Appl. Geophys. 68 (1967), 131-161.

[31] G. P. Williams and D. R. Davies, A mean motion model of the general circulation, Q.J. Roy. Meteorol. Soc. 91 (1965), 471-489.

(Received 1st July 1971) 